Barataeff: Einwirk. eines Gemisches von Jodallyl etc. 7

\title{
34. Einwirkung eines Gemisches von Jodallyl, Jodäthyl und Zink auf Oxalsäureäthyläther;
}

von

\section{S. Barataeff.}

Bei der Einwirkung eines Gemisches von Jodallyl, Jodäthyl und Zink auf Oxalsäureäthyläther konnte ein zweifacher Ausgang der Reaction erwartet werden: entweder reagiren die erwähnten Jodüre zusammen auf den Oxalsäureäther und es bildet sich auf solche Weise bei dieser Reaction Allyläthyloxalsäureäthyläther, ebenso wie Frankland und Duppa ${ }^{1}$ bei der Einwirkung von Jodäthyl mit Jodmethyl Methyläthyloxalsäureäther erhalten haben; oder aber konnte entsprechend den Beobachtungen von J. Kanonnikoff und A. Saytzeff ${ }^{2}$ ) über die Einwirkung der erwähnten Jodüre und Zink auf Ameisensäureäther jedes der Jodüre mit Oxalsäureäther ganz selbständig reagiren, und so ein Gemisch von Diallyl- und Diäthyloxalsäureäther erhalten werden. Auch konnte die Reaction nach beiden Richtungen gleichzeitig vor sich gehen.

Zur Erledigung dieser Frage wurde von mir die folgende Untersuchung ausgeführt.

Ein Gemisch von $1 \mathrm{Mol}$. Oxalsäureäthyläther, $1 \mathrm{Mol}$. Jodallyl und $2 \mathrm{Mol}$. Jodäthyl wurde tropfenweise in eine Retorte, welche gut getrocknetes, feingranulirtes Zink enthielt, eingeführt, wobei die Retorte durch Wasser und Eis abgekühlt wurde. Nach Zufügen des ganzen Gemisches wurde der Retorteninhalt 24 Stunden lang ruhig stehen gelassen, und darauf die mit einem Rückflusskühler versehene Retorte auf dem Wasserbade 10 Stunden lang erwärmt, danach das Produkt mit Wasser zersetzt, wobei eine unbedeutende Erwärmung und Gasentwicklung eintrat. Nach Zusatz von $\mathrm{H}_{2} \mathrm{SO}_{4}$, um Zinkoxyd aufzulösen, und Destillation war ein Oel übergegangen, welches von der wässrigen Flüssig-

\footnotetext{
1) Ann. Chem. 135, 36.

2) Das. 185, 148.
} 
8 Barataeff: Einwirkung eines Gemisches von Jodallyl,

keit getrennt wurde, welche letztere an Aether noch mehr von dem Oel abgab. Die wässrige Flüssigkeit, aus welcher das Oel extrahirt war, wurde in eine Retorte gegossen, von Neuem abdestillirt, und das im Destillate erhaltene Oel auf oben angegebene Weise getrennt. Auf solche Art wurden in zwei Versuchen $326 \mathrm{Grm}$. des rohen Oeles dargestellt, wobei jedesmal $200 \mathrm{Grm}$. Oxalsäureäther, $232 \mathrm{Grm} . \mathrm{C}_{3} \mathrm{H}_{5} \mathrm{~J}$ und $428 \mathrm{Grm} . \mathrm{C}_{2} \mathrm{H}_{5} \mathrm{~J}$ in Arbeit genommen worden waren. Bei der Destillation gerieth das Oel bei ca. $30^{\circ}$ ins Sieden, bei $100^{\circ}$ stieg das Quecksilber ziemlich langsam, von $100^{\circ}$ bis $150^{\circ}$ sehr rasch und von $150^{\circ}$ bis $270^{\circ}$ aufs Neue sehr langsam. Dnrch diese erste Destillation wurde das Oel in folgende Fractionen getheilt: a) von $30^{\circ}$ bis $100^{\circ}$, b) von $100^{\circ}$ bis $150^{\circ}$, c) von $150^{\circ}$ bis $183^{\circ}$, d) von $183^{\circ}$ bis $195^{\circ}$, e) von $195^{\circ}$ bis $210^{\circ}$ und $f$ ) von $210^{\circ}$ bis $270^{\circ}$. Der geringe harzige Rückstand zersetzte sich über $270^{\circ}$. Alle Fractionen wurden (ausser der ersten, welche zwischen $30^{\circ}$ bis $100^{\circ}$ gesammelt wurde und ein Gemisch von Aether, $\mathrm{C}_{2} \mathrm{H}_{6} \mathrm{~J}^{\prime}$ und Diallyl darstellte) der fractionirten Destillation unterworfen. Als Endresultat dieser fractionirten Destillation wurden folgende Fractionen erbalten: a) von $100^{\circ}$ bis $170^{\circ}(2 \mathrm{Grm}$.), b) von $170^{\circ}$ bis $180^{\circ}\left(80 \mathrm{Grm}\right.$.), c) von $180^{\circ}$ bis $185^{\circ}(3 \mathrm{Grm}$.), d) von $185^{\circ}$ bis $190^{\circ}\left(20 \mathrm{Grm}\right.$.), e) von $190^{\circ}$ bis $195^{\circ}(9 \mathrm{Grm}$.), f) von $195^{\circ}$ bis $205^{\circ}\left(1 \mathrm{Grm}\right.$.) und g) von $205^{\circ}$ bis $225^{\circ}(82 \mathrm{Grm}$.)

Nach dem Siedepunkt zu urtheilen, musste der Diäthyloxalsäureäther, dessen Kochpunkt bei $175^{\circ}$ liegt, hauptsächlich sich in der Fraction vom Siedepunkt $170^{\circ}$ bis $180^{\circ}$ befinden; in den Fractionen $195^{\circ}$ bis $205^{\circ}$ und $205^{\circ}$ bis $225^{\circ}$ dagegen der Diallyloxalsäureäther (Siedepunkt bei $213,6^{\circ}$ ). Diese Voraussetzung bestätigte sich auch in der That, da aus den ersten zwei Fractionen eine ziemlich bedeutende Menge einer Säure vom Schmelzpunkt $75^{\circ}$ bis $80^{\circ}$ erhalten wurde, welche auch nach ihren weiteren Eigenschaften und der Analyse des Baryumsalzes als Diäthyloxalsäure sich auswies; aus den letzten zwei Fractionen wurde hauptsächlich eine Säure ausgeschieden, welche nach ihren Eigenschaften und der Analyse des Bleisalzes Diallyoxalsäure war.

Analysen der erwähnten Baryum- und Bleisalze: 
0,2005 Grm. des bei $120^{\circ}$ getrockneten Baryumsalzes gaben $0,1180 \mathrm{Grm} . \mathrm{BaSO}_{4}$, entsprechend $34,6 \% \mathrm{Ba}$, während diäthyloxalsaures Baryum $34,33 \%$ Ba enthält.

$0,3110 \mathrm{Grm}$. des im Exsiccator getrockneten Bleisalzes gaben $0,1695 \mathrm{Grm} . \mathrm{PbSO}_{4}$, entsprechend $37,23 \% \mathrm{~Pb}$. Berechnet für:

$$
\left.\left(\begin{array}{l}
\mathrm{C}_{3} \mathrm{H}_{6} \\
\mathrm{C}_{3} \mathrm{H}_{5}
\end{array}\right\} \text { C. OH . COO }\right)_{2} \mathrm{~Pb}+2 \mathrm{H}_{2} \mathrm{O} 37,43 \% \mathrm{~Pb} \text {. }
$$

Der Allyläthyloxalsäureäther, falls er bei dieser Reaction sich bildet, musste sich, nach seinem muthmasslichen Siedepunkte, in den Fractionen mit dem Siedepunkt $185^{\circ}$ bis $190^{\circ}$ und $190^{\circ}$ bis $195^{\circ}$ befinden, weshalb mit der ersten, in grösserer Quantität erhaltenen Fraction eine Analyse vorgenommen wurde, welche folgende Resultate ergab:

1. 0,1080 Grm. der Substanz gaben 0,2420 Grm. $\mathrm{CO}_{2}$ und 0,0885 Grm. $\mathrm{H}_{2} \mathrm{O}$.

2. 0,2140 Grm. der Substanz gaben 0,4870 Grm. $\mathrm{CO}_{2}$ und $0,1680 \mathrm{Grm} . \mathrm{H}_{2} \mathrm{O}$.

3. 0,2400 Grm. der Substanz gaben 0,5460 Grm. $\mathrm{CO}_{2}$ und 0,1990 Grm. $\mathrm{H}_{2} \mathrm{O}$.

$\begin{array}{crrr} & 1 . & 2 . & 3 . \\ \mathrm{C} & 61,11 & 62,06 & 62,04 \\ \mathrm{H} & 9,10 & 8,72 & 9,21\end{array}$

Berechnet für:

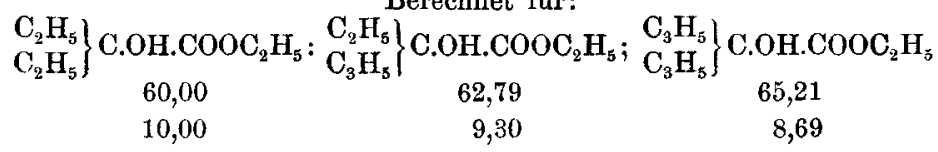

Da diese Analysen nun anzeigten, dass die Substanz noch nicht völlig rein war, so wurde sie aufs Neue fractionirt. Folgende Fractionen: a) $182^{\circ}$ bis $186^{\circ}$, b) $186^{\circ}$ bis $189^{\circ}$ und c) $189^{\circ}$ bis $192^{\circ}$ wurden gesammelt.

Die mittlere, in grösserer Quantität gewonnene Fraction, wurde analysirt:

1. 0,2380 Grm. der Substanz gaben 0,5430 Grm. $\mathrm{CO}_{2}$ und $0,1990 \mathrm{Grm} . \mathrm{H}_{2} \mathrm{O}$.

2. $0,2355 \mathrm{Grm}$. der Substanz gaben $0,5390 \mathrm{Grm} . \mathrm{CO}_{2}$ und $0,1985 \mathrm{Grm} . \mathrm{H}_{3} \mathrm{O}$.

\begin{tabular}{|c|c|c|c|}
\hline \multicolumn{3}{|c|}{ Gefunden: } & Berechnet für: \\
\hline & 1. & 2. & $\left.\mathrm{C}_{3} \mathrm{H}_{5}\right\}$ C. OH. $\mathrm{COOC}_{2} \mathrm{H}_{3}$ \\
\hline $\mathrm{C}$ & 62,62 & 62,42 & 62,79 \\
\hline $\mathbf{H}$ & 9,29 & 9,37 & 9,30 \\
\hline
\end{tabular}


10 Barata eff: Einwirkung eines Gemisches von Jodallyl,

Obgleich die analysirte Substanz die Zusammensetzung des Allyläthyloxalsäureäthyläthers besitzt, so konnte sie dennoch ein Gemenge gleicher Mol. von Diäthyl- und Diallyloxalsäureäther sein. Diese Frage konnte nur ein weiteres Studium der Säure selbst und ihrer Salze entscheiden. Zur Darstellung von Salzen dienten Fractionen des Aethers, welche bei der dem Siedepunkte des analysirten Produktes nahen Temperatur überdestillirt waren. Die Fractionen $190^{\circ}$ bis $195^{\circ}$ und $189^{\circ}$ bis $192^{\circ}$, und auch $182^{\circ}$ bis $186^{\circ}$ wurden mit alkoholischem Kali verseift und aus den, durch $\mathrm{H}_{2} \mathrm{SO}_{4}$ freigemachten und mit Aether extrahirten Säuren die Calcium-, Baryum- und Zinksalze dargestellt.

Das Calciumsalz scheidet sich im Exsiccator aus der wässrigen Lösung in Gestalt eines dünnen Häutchens auf der Oberfläche $a b$, welches zu Boden fällt und einem neuen Häutchen Platz macht. Das auf solche Weise abgeschiedene $\mathrm{Salz}$ wurde von der Mutterlauge getrennt, und letztere bis zur Trockne abgedampft. Das in Gestalt eines Häutchens abgeschiedene und das aus der Mutterlauge erbaltene Salz wurden besonders gesammelt und analysirt.

1. 0,2135 Grm. des in Gestalt eines Häutchens abgeschiedenen und bei $120^{\circ}$ getrockneten Salzes gaben $0,0860 \mathrm{Grm}$. $\mathrm{CaSO}_{4}$.

2. $0,2710 \mathrm{Grm}$. des aus Mutterlange erhaltenen und bei $120^{\circ}$ getrockneten Salzes gaben $0,1140 \mathrm{Grm}$. $\mathrm{CaSO}_{4}$.

1. Gefunden:

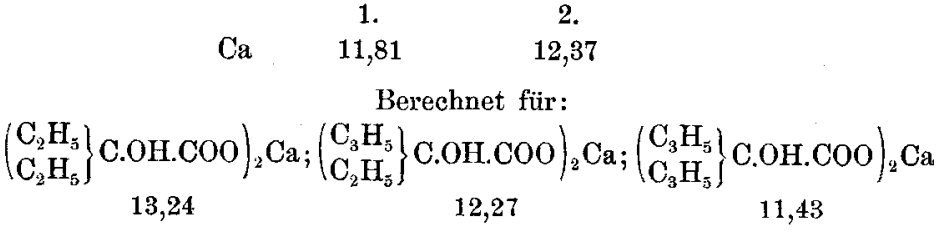

Das Baryumsalz, aus wässriger Lösung abgeschieden, ist dem äusseren Ansehen nach dem Calciumsalz sehr ähnlich; aber, da es in Wasser löslicher ist, so scheidet es sich aus der Lösung schwerer, als das letztere, ab. Dieses Salz wurde zur Analyse ebenso, wie das Calciumsalz getrennt.

Ausserdem wurde auch noch ein Baryumsalz einer Analyse unterworfen, welches aus der Säure gewonnen, die in geringer Menge in Gestalt eines Baryumsalzes im Niederschlag (zusammen mit $\mathbf{B a C O}_{3}$ ) 


\section{Jodäthyl und Zink auf 0xalsäureäthyläther. 11}

nach der Darstellung der vorhergehenden, aus der wässrigen Lösung abgeschiedenen Baryumsalze, zurückgeblieben war.

Analyse des bei $120^{\circ}$ getrockneten Baryumsalzes:

1. 0,2545 Grm. des aus wässriger Lösung in Gestalt eines Häutchens abgeschiedenen Salzes gaben $0,1350 \mathrm{Grm} . \mathrm{BaSO}_{4}$.

2. $0,3630 \mathrm{Grm}$. desselben Salzes gaben 0,1915 Grm. $\mathrm{BaSO}_{4}$.

3. 0,2015 Grm. des, aus der als Baryumsalz im Niederschlag, zusammen mit $\mathrm{BaCO}_{3}$, zurückgeblicbenen Säure dargestellten Salzes gaben 0,1070 Grm. $\mathrm{BaSO}_{4}$.

4. 0,1985 Grm, desselben Salzes gaben 0,1075 Grm. $\mathrm{BaSO}_{4}$.

5. 0,2960 Grm. des durch Abdampfen der Mutterlauge bis zur Trockne gewonnenen Salzes gaben $0,1630 \mathrm{Grm} . \mathrm{BaSO}_{4}$.

6. $0,5010 \mathrm{Grm}$. desselben Salzes gaben 0,2730 Grm. $\mathrm{BaSO}_{4}$.

Gefunden:

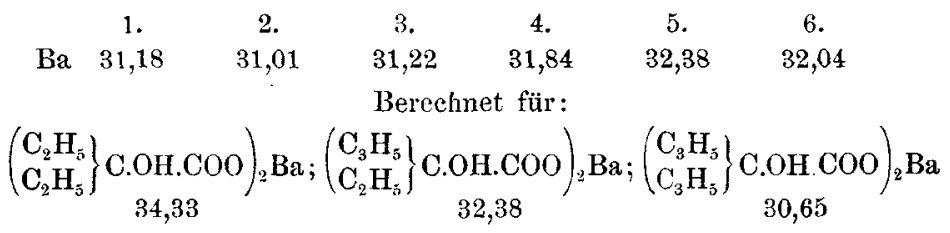

Das Zinksalz schied sich aus wässriger Lösung ähnlich den vorhergehenden Salzen in Form eines Häutchens $a b$, wurde von der Mutterlauge getrennt, und die letztere zur Trockne abgedampft.

Analyse des bei $120^{\circ}$ getrockneten Salzes :

1. 0,2115 Grm. des in Gestalt eines Häutchens abgeschiedenen Salzes gaben 0,0465 Grm. ZnO.

2. $0,1510 \mathrm{Grm}$. desselben Salzes gaben 0,0335 Grm. ZnO.

3. 0,1745 Grm. des aus der Mutterlauge gewonnenen Salzes gaben $0,0425 \mathrm{Grm}$. $\mathrm{ZnO}$.

4. 0,1435 Grm. desselben Salzes gaben 0,0345 Grm. ZnO. Gefunden :

\begin{tabular}{|c|c|c|}
\hline $\begin{array}{c}1 . \\
17,64\end{array}$ & $\begin{array}{c}2 . \\
17,80\end{array}$ & $\begin{array}{c}3 . \\
19,54\end{array}$ \\
\hline
\end{tabular}

Berechnet für :

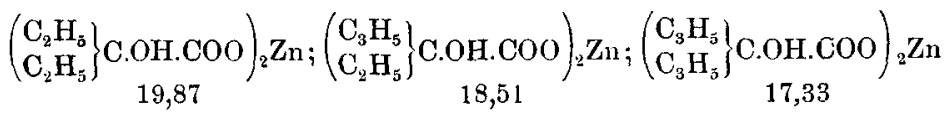

Die nach der Zersetzung des von den Analysen übrig gebliebenen Baryumsalzes erhaltene freie Säure schied sich aus einer wässrigen Lösung in zwei scharf von einander verschiedenen Formen ab: in seideartigen, farblosen, sehr 
12 Barataeff: Einwirkung eines Gemisches von Jodallyl, dünnen Nadeln und in fast undurchsichtigen, warzigen Aggregaten. Diese Krystalle wurden von einander mechanisch getrennt, und darauf einige neuen Krystallisationen unterworfen, wobei jedesmal die Krystalle der einen Form von denen der anderen mechanisch getrennt wurden. Endlich wurde aus den Krystallen der ersten Form eine Säure vom Schmelzpunkte und den Eigenschaften der Diätbyloxalsäure, und aus den Krystallen der zweiten Form Diallyloxalsäure erhalten.

Zieht man die oben erwähnten Resultate der Untersuchung in Betracht, so gelangt man zu dem Schlusse, dass die, aus den Fractionen des Aethers von den Siedepunkten $189^{\circ}$ bis $192^{\circ}$ erhaltene Säure ein Gemenge von Diäthylund Diallyloxalsäure ist, worin die letztere den Haupttheil bildet. Jene Schlussfolgerung, dass die untersuchte Säure ein Gemisch von Diäthyl- und Diallyloxalsäure und nicht Allyläthyloxalsäure darstellt, bekräftigen: 1. die zweifache Krystallform der durch Zersetzung des Baryumsalzes erhaltenen Säuren, 2. der verschiedene Sehmelzpunkt und die sonstige Verschiedenheit der nadelförmigen und warzigen Krystalle und endlich 3. die, bei den Analysen der Calcium-, Baryum- und Zinksalze gewonnenen Resultate. Die aus wässriger Lösung abgeschiedenen und von der Mutterlauge getrennten Salze lieferten Zahlen, welche denen nahe kommen, die der Menge des $\mathrm{Ba}, \mathrm{Ca}$ und $\mathrm{Zn}$ in den Salzen der Diallyloxalsäure anzeigen, während die Analysen der, durch Abdampfen der Mutterlauge dargestellten Salze auf die Zusammensetzung der diäthyloxalsauren Salze hinweisen. Eine solche Erscheinung ist ganz verständlich für eine Mischung der erwähnten Säuren, da die Salze dieser Säuren durch ihre Löslichkeit in Wasser sich von einander im bedeutenden Grade unterscheiden. Die Salze der Diallyloxalsäure lösen sich in Wasser schwerer, als die der Diäthyloxalsäure. Die Analysen sowohl der von der Mutterlauge getrennten, als auch der beim Abdampfen derselben gewonnenen Salze bestätigen diese Voraussetzung vollständig, Je grösser der Unterschied in der Löslichkeit dieser Salze, desto vollkommener gelingt ihre Trennung. Dieses ist aus den Resultaten 
der Analyse des Zinksalzes ersichtlich: das diallyloxalsaure Zink löst sich sehr schwer in Wasser (nach Beobachtungen von E. Schatzky sind in 100 Thln. der bei $22^{\circ}$ gesättigten Lösung 0,22 Thl. des wasserfreien Salzes enthalten), deshalb scheidet es sich auch aus der Lösung bereits fast ganz aus, ehe noch das weit leichter in Wasser lösliche diäthyloxalsaure Zink sich abzuscheiden beginnt. Dagegen gelingt die Trennung der $\mathrm{Ba}$ - und Ca-Salze, welche nicht so scharf durch das Löslichkeitsverhältniss unterschieden sind, minder vollkommen. Aber bei alledem weisen die Analysen dieser letzteren Salze mit hinreichender Deutlichkeit darauf hin, dass in den sich früher abscheidenden Salzen die Diallyloxalsäure, in den aus den Mutterlaugen gewonnenen Salzen dagegen die Diäthyloxalsäure vorwiegt. Der Schluss, dass in der untersuchten Säure die Diallyloxalsäure verhältnissmässig vorherrscht, findet Bestätigung nicht nur in dem Siedepunkte der untersuchten Fraction des Aethers, sondern auch in den Resultaten, welche bei der Analyse des aus der Mutterlauge gewonnenen $\mathrm{Ba}$ - und $\mathrm{Ca}$-Salzes erhalten wurden. Diese Analysen beweisen, dass, obwohl aus der Lösung ziemlich viel von diallyloxalsaurem Calcium oder Baryum abgeschieden worden, dennoch in der Lösung eine bedeutende Menge dieses Salzes übrig bleibt.

Untersuchung deraus der Fraction des Aethers mit dem Siedepunkte $182^{\circ}-186^{\circ}$ gewonnenen Säure. - Die aus dieser Fraction gewonnene Säure wurde in das Baryumsalz verwandelt. Dasselbe schied sich aus wässriger Lösung in Gestalt eines Häutchens $a b$, welches von der Mutterlauge abfiltrirt wurde; diese letztere wurde sodann zur Trockne abgedampft. Die Analysen der auf solche Weise gewonnenen und bei $120^{\circ}$ getrockneten Salze lieferten folgende Resultate:

1. 0,3378 Grm. des in Gestalt eines Häutchens abgeschicdenen Salzes gaben $0,1 \mathrm{~s} 90 \mathrm{Grm}$. $\mathrm{BaSO}_{4}$.

2. 0,1455 Grm. desselben Salzes gaben 0,0830 Grm. BaSO .

3. 0,4055 Grm. des beim Abdampfen der Mutterlauge erhaltenen Salzes gaben 0,2300 Grm. $\mathrm{BaSO}_{4}$.

4. $0,4000 \mathrm{Grm}$. dessclben Salzes gaben 0,2280 Grm. $\mathrm{BaSO}_{4}$. 
14 Barataeff: Finwirkung eines Gemisches ron Jodallyl,

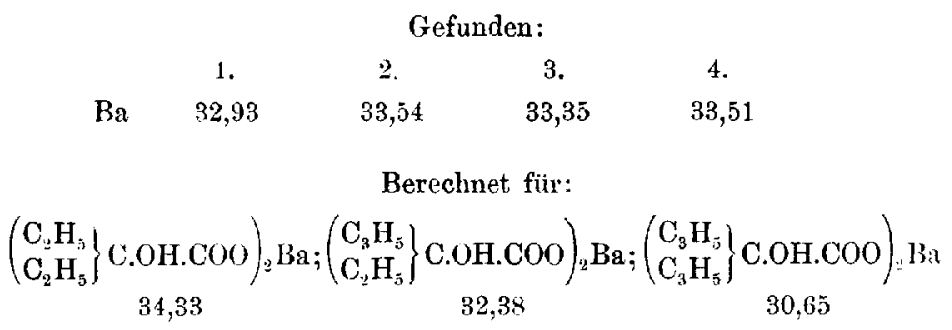

Die aus dem von den Analysen übrig gebliebenen Salzı. abgeschiedene Säure krystallisirte in zwei Formen: in nadelförmigen Krystallen und in Warzen. Nach etlichen Krystallisationen und mechanischer Trennung der verschiedenen Krystalle bis man völlig gleichartige erhielt, schmolzen die nadelförmigen Krystalle bei $74^{\circ}$ bis $76^{\circ}$ und erstarrten bei $72^{\circ}$ bis $70,5^{\circ}$, die warzigen aber schmolzen bei $48^{\circ}$ bis $50^{\prime \prime}$ und erstarrten bei $46^{\circ}$ bis $44,5^{\circ}$. Daraus folgt, dass die aus der Fraction vom Siedepunkt $182^{\circ}$ bis $186^{\circ}$ gewonnene Säure gleichfalls ein Gemisch von Diäthyl- und Diallyloxalsäure darstellt, von denen die erstere in der Mischung vorherrscht. $\mathrm{Zu}$ Gunsten dieser letzteren Annahme sprechen auch die Analysen des Baryumsalzes, da der bei allen diesen Analysen crhaltene Procentgchalt an Baryum sich mehr der Zusammensetzung des diäthyloxalsauren Salzes nähert, als der des diallyloxalsauren.

Untersuchung der Säure aus der Fraction vom Siedepunkt $186^{\circ}$ bis $189^{\circ}$. Auf Grund der oben erwähnten Analyse dieser Fraction des Aethers musste mau erwarten, dass sie entweder Allyläthyloxalsäureäthyläther, oder ein Gemisch von Diäthyl- und Diallyloxalsäureäthyläther nach gleichen Mol. darstellte. Diese Voraussetzung bestätigte auch die Analyse der aus dieser Fraction des Aethers freigemachten Säure. Der Aether wurde mit alkoholischer Aetzkalilösung zersetzt, der Alkohol abdestillirt. das Kaliumsalz mit $\mathrm{H}_{2} \mathrm{SO}_{4}$ zersetzt und zuletzt die freigewordene Säure mit Aether extrahirt. Vor der Aualyse wurde die Säure im Exsiccator getrocknet. 
Jodäthyl und Zink auf Oxalsäureäthyläther. 15

$0,2215 \mathrm{Grm}$. der Säure gaben $0,4760 \mathrm{Grm}$. $\mathrm{CO}_{2}$ und $0,1700 \mathrm{Grm} . \mathrm{H}_{2} \mathrm{O}$.

$\begin{array}{cc} & \text { Gefunden: } \\ \mathrm{C} & 58,60 \\ \mathrm{H} & 8,53\end{array}$

Berechnet für:

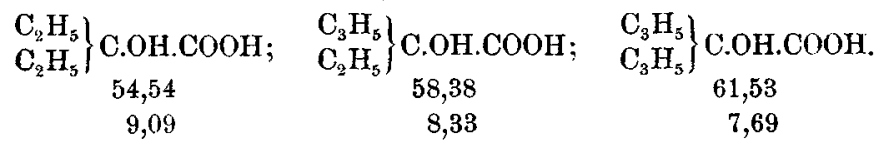

Das Baryumsalz dieser Säure schied sich aus der wässrigen Lösung ähnlich den oben erwähnten Baryumsalzen ab. Zur Analyse wurde aus der zuvor stark eingeengten Lösung abgeschiedenes Salz angewandt. $\mathrm{BaSO}_{4}$.

1. 0,2405 Grm. des bei $120^{\circ}$ getrockneten Salzes gaben 0,1315 Grm. $\mathrm{BaSO}_{4}$.

2. $0,1505 \mathrm{Grm}$, des bei $120^{\circ}$ getrockneten Salzes gaben 0,0825 Grm.

Gefunden:

$\mathrm{Ba}$

$32,15 \quad 32,23$

Berechnet für:

$\left(\begin{array}{l}\mathrm{C}_{2} \mathrm{H}_{5} \\ \mathrm{C}_{2} \mathrm{H}_{5}\end{array}\right\}$ C.OH.COO $)_{2} \mathrm{Ba} ;\left(\begin{array}{l}\mathrm{C}_{3} \mathrm{H}_{5} \\ \mathrm{C}_{2} \mathrm{H}_{5}\end{array}\right\}$ C.OH.COO $)_{2} \mathrm{Ba} ;\left(\begin{array}{l}\mathrm{C}_{3} \mathrm{H}_{5} \\ \mathrm{C}_{3} \mathrm{H}_{5}\end{array}\right\}$ C.OH.COO $)_{2} \mathrm{Ba}$ 34,33

32,38 30,68

Die Säure, welche aus dem von der Analyse zurückgebliebenen Baryumsalze dargestellt worden war, schied sich aus der wässrigen Lösung in derselben doppelten Form ab, wie auch die aus den oben erwähnten Fractionen des Aethers gewonnene Säure. Die nadelförmigen, von den Warzen getrennten Krystalle wurden einige Male aus Wasser umkrystallisirt, wobei man die ersteren jedesmal von den Warzen mechanisch trennte. Die auf solche Weise erhaltenen völlig gleichartigen Krystalle schmolzen bei $74,5^{\circ}$ bis $75,5^{\circ}$ und erstarrten bei $72^{\circ}$ bis $74^{\circ}$. Obwohl dieser Schmelzpunkt der Diäthyloxalsäure angehört, wurde doch zum Ueberfluss das Baryumsalz der Säure dargestellt, welches bei der Analyse folgende Resultate lieferte. $\mathrm{BaSO}_{4}$.

1. $0,2510 \mathrm{Grm}$. des bei $120^{\circ}$ getrockneten Salzes gaben $0,1470 \mathrm{Grm}$. $\mathrm{BaSO}_{4}$.

2. $0,1065 \mathrm{Grm}$. des bei $120^{\circ}$ getrockneten Salzes gaben $0,0620 \mathrm{Gm}$. 
16 Barataeff: Einwirk. eines Gemisches von Jodallyl etc.

\begin{tabular}{cccc} 
& \multicolumn{2}{c}{ Gefunden: } & Berechnet für: \\
& 1. & 2. & $\left(\begin{array}{c}\mathrm{C}_{2} \mathrm{H}_{5} \\
\mathrm{C}_{2} \mathrm{H}_{5}\end{array}\right)$ C.OH.COO $)_{2} \mathrm{Ba}$ \\
$\mathrm{Ba}$ & 34,43 & 34,24 &
\end{tabular}

Die in Gestalt von Warzen ausgeschiedene Säure wurde nach der Trennung von den nadelförmigen Krystallen einige Mal aus Wasser umkrystallisirt, wobei man die Nadeln jedesmal mit Sorgfalt entfernte. Nachdem völlig gleichartige Krystalle erhalten worden waren, erwiesen sie sich dem äusseren Ansehen nach der Diallyloxalsäure äusserst ähnlich, hatten auch den Schmelzpunkt der letzteren: $48^{\circ}$ bis $49,5^{\circ}$, und erstarrten bei $46^{\circ}$ bis $45^{\circ}$. Das aus diesen Krystallen dargestellte und im Exsiccator getrocknete Bleisalz lieferte bei der Analyse folgendes Resultat:

$0,2715 \mathrm{Grm}$. des Salzes gaben 0,1475 Grm. $\mathrm{PbSO}_{4}$.

Gefunden:

Berechnet für:

$\mathrm{Pb} \quad 37,12$

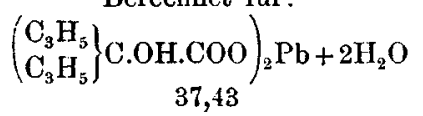

Aus allen diesen bei der Untersuchung der Fraction des Aethers vom Siedepunkt $186^{\circ}$ bis $189^{\circ}$ erhaltenen Daten geht ohne Zweifel hervor, dass dieser Aether ein Gemisch von Diäthyl- und Diallyloxalsäureäthyläthern nach gleichen Mol. ist.. Wie es mir scheint, kann man es also für bewiesen erachten, dass durch Einwirkung von $\mathrm{C}_{2} \mathrm{H}_{5} \mathrm{~J}, \mathrm{C}_{3} \mathrm{H}_{5} \mathrm{~J}$ und Zink auf Oxalsäureäthyläther nicht der Allyläthyloxalsäureäthyläther, sondern ein Gemisch von Diäthyl - und Diallyloxalsäureäther sich bildet. - In dem gegebenen Falle geht folglich die Reaction ganz analog derjenigen vor sich, welche von Prof. A. Saytzeff in Gemeinschaft mit J. Kanonnikoff für den Ameisensäureäther bei der Einwirkung derselben Jodüre in Gegenwart von Zink nachgewiesen ist. 